\title{
COMPETENCIAS DOCENTES: DE LA PRÁCTICA REFLEXIVA AL CAMBIO DEL HABITUS ACADÉMICO
}

\author{
Karina Rodríguez Cortés *
}

\begin{abstract}
RESUMEN
En la ponencia se analizan las competencias docentes desde la mirada teórica de Phillippe Perrenoud, quien comparte con Pierre Bourdieu, la explicación proposi- cional de lo social y lo educativo. El trabajo está dividido en tres apartados: en el primero, presentamos el significado de la acción social; en el segundo, la práctica educativa como campo reproductor de significados; y en el tercero, la práctica reflexiva como base del desarrollo de las competencias docentes. Para concluir se integra un conjunto de reflexiones que pretenden ubicar uno de los puntos críticos relacionados con el tema, esto es la modificación del habitus del homo academicus.
\end{abstract}

\section{ABSTRAT}

In the conference, educational competences are analyzed from the theoretical glance of Phillippe Perrenoud, who shares with Pierre Bourdieu, the propositional explanation of the social and the educational. The paper is divided into three sections: in the first, we presented the meaning of social action; in the second, the educational practice as reproductive field of meanings; and in the third sec- tion, the reflective practice as base of the development of the educational compe-tences. In conclusion, a set of reflections is integrated which try to locate one of the critical points related to the topic, this is the modification of habitus of homo academicus

\footnotetext{
* Candidata a Doctora en Ciencias Sociales, con especialidad en Educación y Sociedad por la UAM-Xochimilco. Maestra en Investigación y Desarrollo de la Educación por la Univer- sidad Iberoamericana. Licenciada en Pedagogía por la Universidad Pedagógica Nacional. Ha sido docente en licenciaturas, diplomados y maestrías en diferentes instituciones de educación superior en México. ad0 2037@ulsa.mx
} 


\section{INTRODUCCIÓN}

Una de las críticas realizadas al tema de competencias en México, es la relacionada con la carencia de un respaldo teórico sólido, en el terreno de las ciencias sociales o en el campo de la educación. En términos generales podemos ubicar tres referentes teóricos que están explican- do el tópico con la finalidad de transformar las prácticas educativas y los espacios sociales concretos, éstos son: el conjunto de autores que clasifican y analizan las operaciones y las funciones mentales (Jean Pia- get, Leontiev Seminovich Vigostky, Ruven Fourestein, entre otros); en la sociología, Phillippe Perrenoud, desde la perspectiva de Pierre Boudieu, explica la educación como una acción social con fines determinados ("la educación no se puede entender en sí misma"); y en la filosofía social, Jürgen Habermas argumenta a partir de la acción comunicativa la posi- bilidad de la transformación social basada en la ética.

En la presente ponencia pretendemos analizar las competencias docen- tes desde el referente de Phillippe Perrenoud, quien comparte con Pie- rre Bourdieu, la explicación en torno a que las instituciones educativas están organizadas para mediar los valores, las normas y las actitudes congruentes y en conformidad con los valores de cada sociedad con- creta. En un primer apartado presentamos el significado de la acción social; en un segundo, la práctica educativa como campo reproductor de significados; y en el tercero, la práctica reflexiva como base del desarrollo de las competencias docentes. Para concluir se presenta un conjunto de reflexiones que pretenden ubicar los puntos críticos sobre las competencias docentes.

\section{La acción social como base de prácticas educativas liberadoras}

La fórmula para la determinación de la acción social desde la perspec- tiva de Pierre Bourdieu, asentada en la razones prácticas atraviesa las posiciones sociales (concepto relacional), las disposiciones (o los habitus) y las tomas de posición (las elecciones) que los agentes sociales llevan a cabo en los diversos ámbitos de la práctica. Iniciamos por las proposi- ciones de: espacio y espacio social (campo). La primera, la define como un conjunto de posiciones distintas y coexistentes, externas unas a otras, caracterizadas en relación unas de otras, por su exterioridad mu- tua y por las relaciones de proximidad y asimismo por las relaciones de orden. La segunda, como una aprehensión relacional del mundo social: "afirma en efecto que toda la realidad que designa, reside en la exterio- ridad mutua de los elementos que la componen". El habitus cumple una función que, en otra filosofía, se confía a la conciencia transcendente: 
"es un cuerpo socializado, un cuerpo estructurado, un cuerpo que se ha incorporado a las estructuras inmanentes de un mundo o de un sector particular de este mundo, de un campo, y que estructura la percepción de este mundo y también de la acción en este mundo" (Bourdieu, 997:46).

Los seres aparentes, directamente visibles, trátese de individuos o de grupos, "existen y subsisten en y por la diferencia, es decir en tanto que ocupan posiciones relativas en un espacio de relaciones que aunque invisible y siempre difícil de manifestar empíricamente, es la realidad más real y el principio real de los comportamientos de los individuos y de los grupos" (Bourdieu, 997: 47). El espacio social es un campo de fuer- zas, que se impone a los agentes que se han adentrado en él, y como un campo de luchas dentro del cual los sujetos se enfrentan, con medios y fines diferenciados, según su posición en la estructura del campo de fuerzas. La constitución o consagración simbólica de un campo es necesaria para crear un grupo unido "(imposición de nombres, de siglas, de signos de adhesión, manifestaciones públicas, etc)", que tiene posibilidades varias de alcanzar el éxito en cuanto que los agentes sociales sobre los que se ejerce estén más propensos, debido a su proximidad en el espacio de la posiciones sociales y también de las disposiciones y de los intereses asociados a determinadas posiciones.

La acotación del campo permite inferir que existen varios campos, Claudette Lafaye en La sociología de las organizaciones, resume el concepto de campo en la sociología de Bourdieu en los siguientes términos:

Un campo es un espacio estructurado - por lo tanto, jerárquico- que incorpora posiciones o puestos cuyas características son relativamente independientes de quienes los ocupan.

- Cada campo se define en términos de sus retos e intereses específicos comparados con los de otro campo; esto significa, por ejemplo, que un científico está interesado en cosas diferentes de las que le interesan a un hombre de negocios o de la iglesia.

Un campo también implica la posesión o constitución de un capital específico. Un capital económico grande es fundamental en el mundo de los negocios pero incongruente con el campo científico, en el que el capital relevante es de una naturaleza distinta: una tesis, publicaciones con prestigio, reconocimiento internacional, etc. 
- Un campo necesita de agentes sociales con disposiciones adecuadas -lo que Bourdieu llama habitus_ que puedan actuar en él, lo cual implica el conocimiento de la reglas del juego del campo en cuestión.

'La estructura de un campo es el resultado, en un tiempo t, del equilibrio del poder entre los agentes o instituciones que ocupan distintas posiciones.

-Un campo también es un espacio dinámico en que se llevan a cabo batallas por la conservación o cambio equilibrado del poder: ocupar posiciones dominantes, cambiar de una posición de servicio a una dominante, estabilizar posiciones precarias, obtener reconocimiento de las posiciones de los márgenes de un campo, descalificar otras, etc.

-Un campo no es un espacio cerrado. En especial los márgenes de un campo están constantemente sujetos a luchas de poder entre los agentes u organizaciones que lo conforman.

-Dentro de un campo determinado, la lucha que se da entre quienes ocupan posiciones distintas presupone un acuerdo fundamental en cuanto al motivo de la lucha.

El sentido práctico, el campo es un sistema adquirido de preferencias, de principios de visión y de división (gusto), de estructuras cognitivas y de esquemas de acción que orientan la percepción de la situación y de la respuesta adaptada. Bajo dicha reflexión teórica, el habitus "es una especie de sentido práctico de qué hay que hacer en una situación determinada" (Bourdieu, 997: 40), lo que orienta a los sujetos hacia la toma de decisiones ordenadas según el orden objetivo. Cumple con una función que “... se confía a la consciencia trascendente: es un cuerpo socializado, un cuerpo estructurado que se ha incorporado a las estructuras inmanentes de un mundo o de un sector particular de este mundo, de un campo, y que estructura la percepción de este mundo y también la acción en este mundo" (Bourdieu, 997: 46).

Significa que el espacio de las posiciones sociales se traduce en un espacio de toma de posición a través del espacio de las disposiciones o del sistema de desviaciones diferenciales en las propiedades de los agentes (en sus prácticas y en los bienes que poseen). La categoría de campo le permite a Pierre Bourdieu, aseverar que la reproducción de la estructura del capital cultural tiene como base las estrategias de las 
familias y la lógica específica de la institución escolar. Las familias son cuerpos impulsados por una tendencia a perpetuar su ser social, con todos sus poderes y privilegios, las posiciones independientes de los poderes temporales y espirituales establecidos, corren parejas con el desarrollo de una burguesía, cuyos intereses en materia de reproduc ción están estrechamente vinculados con el colegio. La "nueva clase" eleva sus intereses particulares a un grado de universalización superior e inventa una versión que llama "progresista".

Philiphe Perrenoud, en coincidencia con el desarrollo teórico de Bourdieu, subraya que con el proceso de escolarización, y el paso de los años, los individuos participan con frecuencia en varios campos:

-El campo de las relaciones con los padres, en el que cada individuo es, y continúa siendo, hijo de sus padres. Con el paso del tiempo puede comenzar una nueva familia y volverse él mismo padre.

-El campo de la cultura, los valores y las representaciones sociales, por lo menos desde la edad en que uno es capaz de comunicarse y entender.

-El campo de la ley y la justicia: incluso antes de nacer los niños son sujetos con derechos.

•El campo de la religión, si la familia de un individuo es creyente o si el individuo mismo se vuelve creyente.

-El campo de la salud, en cuanto uno depende de la medicina y la seguridad social.

-El campo del consumo, primero a través de los padres y paulatina- mente por cuenta propia.

-El campo político, en cuanto se llega a la edad en la que puede participar o ser sujeto de decisiones.

-El campo de los medios y la información en una sociedad en la que, si no es más que un consumidor, no se escapa de las industrias de cultura y comunicación masiva. 
•En el campo de la comunidad, en cuanto se tiene la edad para unirse al club, asociación, sindicato o partido.

•En el campo del trabajo, primero en caso y después en relaciones la- borales y del trabajo pagado, en ocasiones desde la infancia.

•El campo de la educación y la formación, desde el nacimiento dentro de la familia y después en la escuela desde la edad de dos a cuatro años.

En la jerarquía que caracteriza a los campos, el acto de clasificación escolar es un acto de ordenación en el sentido que instituye una dife- rencia social de rango, son miembros de un orden. Los agentes sociales al optar por una carrera o disciplina, "no son partículas sometidas a fuerzas mecánicas y que actúan bajo la imposición de causas; como tampoco son sujetos conscientes y avezados que obedecen a razones y que actúan con pleno conocimiento de causa como creen los defen- sores de Rational Action Theory" (Bourdieu, 997: 39). La posición del teórico francés es que los sujetos son agentes actuantes y conscientes dotados de un sentido práctico.

Para ser actor en un campo "X", "debe haber una suerte de familiaridad con el conocimiento, valores, reglas, ritos, códigos, conceptos, lengua- je, leyes, instituciones y objetos específicos del campo en cuestión. Por esta razón, entrar en un campo nuevo exige un proceso de socialización cuya duración es variable, en algunos casos está bien organizado, en otros no y por lo general depende de la posición específica dentro del campo" (Simona, 200 :229-230). Esto significa que se necesitan distin- tos tipos de socialización para incursionar en cualquier campo, nos en- contramos con la noción de espacio social, que contiene el principio de una aprehensión relacional del mundo social, que se impone al indivi- duo con medios y fines diferenciados según su posición en la estructura del campo, contribuyendo a conservar o a transformar la estructura.

\section{La práctica educativa como campo reproductor}

El concepto de campo explica cómo la estructura social determina la praxis en un individuo o en un grupo, la sociedad está estructurada por varios campos, en forma de esquemas (casi inconscientes) de per- cepción, de concepción, y de acción que son comunes a un grupo de- terminado. $Y$ también permite entender la práctica educativa como re- productora $y$ conservadora, aceptada como normal y pertinente para ellogro de objetivos individuales y sociales, como una práctica reproductora de cultura. Podemos decir, "que la educación produce significados. La educación no sólo es sino que también significa, produce significa- dos concretos que se suman al conjunto de productos culturales que forman objetivamente el 'espesor' cultural de una sociedad concreta. Tal cultura objetiva, y también los significados, confieren, refuerzan la identidad de la sociedad concreta" (Simona, $200: 2$ ). 
La posibilidad de re-significación no está garantizada por el hecho de que la práctica educativa se realice, ni tampoco que contribuya al pro- yecto de transformación social, se pueden sofisticar y reforzar las prác- ticas conservadoras. Es un hecho que la práctica educativa produce significación y re-significación en ocasiones innovadoras y modificado- ras de la identidad social de una cultura concreta, mediante lo cual se puede contribuir a modificar la estructuración y la modificación de las relaciones sociales. Pierre Bourdieu, permitió regresar a la educación, la posibilidad de vincular la educación con la acción social, proceso que se constituye en educativo cuando se problematiza la acción educativa, no es así, cuando se mide la profesionalización de los docentes universitarios o la tutorías que éstos realizan.

Con base en lo anterior, podemos sostener que la acción educativa es "un conjunto de prácticas que los sujetos educativos constituyen con base en la relación-interacción que establecen y en la cual se suceden percepciones e interpretaciones continuas, las que suscitan nuevas acciones fruto de las interpretaciones sucesivas, dentro del contexto in- fluyente en la percepción y la interpretación, y a la vez 'hermenéutico', susceptible de interpretación. Esa sucesión conforma una continuidad de significaciones, y se conforma así una verdadera lógica de significaciones" (Simona, 200 :24). En síntesis, el estudio de la acción educativa implica el análisis de la interacción entre los sujetos en la cotidianidad- formalidad del proceso educativo, lo que presenta como supuesto, la necesidad de contar con un esquema general de análisis de los procedimientos interpretativos y de la significación.

La interacción está en el plano de la subjetividad y de las vivencias personales, puede ser una experiencia en común entre dos o más personas que proceden con acción lógica a una interpretación. Todos "los seres humanos poseemos un yo y somos reflexivos, es decir, 'interactuamos' con nosotros mismos: pensamos lo que hacemos y lo que ocurre en nuestras mentes es un elemento esencial para de- 
cidir nuestro comportamiento. Ese yo es dinámico, no se detiene, cambia su acción. Los otros y los objetos a los cuales nos enfrenta- mos afectan la 'interacción' con nosotros mismos y nos enfrentan a decisiones acerca de esos otros, o de esos objetos. $Y$ esas decisio- nes no son azarosas o caprichosas, sino fruto de nuestra reflexión, aunque exista la posibilidad de actos 'irreflexivos', 'impulsivos'. Así nos damos cuenta que actuamos de acuerdo con la forma en que interpretamos el mundo que nos rodea" (Simona, 200 :26).

Desde el interaccionismo simbólico, una interacción es un orden nego- ciado frágil, temporal que debe ser construido permanentemente con el fin de interpretar el mundo. Para Max Weber, existe interacción social cuando una persona actúa frente a la otra con la expectativa de que ésta también actúe, o al menos se dé cuenta de la existencia y de la acción desempeñada por el sujeto. Este último requiere que el interlocutor se dé cuenta de la existencia de aquél, e interprete lo que éste hace o dice como indicación de lo que ocurre en la mente de aquél, de ahí puede suscitarse la acción recíproca del interlocutor o no. Alfred Schutz, discí- pulo de Weber, formula la pregunta relacionada con él cómo sucede ese intercambio de subjetividades y cómo se logra precisamente producir el contexto motivacional intersubjetivo.

Para el autor, existen dos actitudes: "una es la de vivir en nuestros actos, estando dirigidos hacia los objetos de ellos, y la otra, la acti- tud reflexiva, por la cual nos dirigimos hacia nuestros actos, captán- dolos por medio de otros actos. "Vivir nuestros actos significa vivir en nuestro presente, en el cual no somos conscientes de nuestro yo ni de nuestro pensamiento. Para alcanzar nuestro sí mismo si no reflexionamos. Pero nuestra reflexión no capta el presente sino el pasado. En otras palabras, la autoconciencia no puede ser experi- mentada sino en tiempo pasado" (Simona, 200 :30).

El supuesto que resume los planteamientos de Schutz, consiste en lo siguiente: "vivimos el mundo, no como mundo privado, sino común a todos; este mundo se nos da a todos y es potencialmente accesible a cada uno". No obstante, cuando dos sujetos observan un mismo hecho, no interpretan necesariamente lo mismo. Al respecto Aarón Cicourel propone una caracterización de los procedimientos interpretativos (pp.

$3-32)$ : 
La reciprocidad de las perspectivas: explica cómo los sujetos en interacción intercambian puntos de vista y forman un sistema de pertinencia.

2.La hipótesis: significa que podemos entender desde el lugar del otro e incluso comprender su punto de vista y no tener una comprensión común necesaria para mantener y hacer productivo el intercambio.

3.Las formas normales: representan las formas de expresión conside- radas como formales, a las cuales los sujetos acuden cuando existe disonancia en el intercambio.

4.El carácter retrospectivo-prospectivo de los acontecimientos: clarifi- car lo dicho con lo que vendrá, o completar con lo dicho previamente, organiza el sentido de lo que se va a decir.

5.El lenguaje es reflexivo: como constitutivo de nuestra vida social, per- mite describir y hacer comprensible las actividades, los escenarios y las intenciones en medio de los cuales se desarrolla la acción, porque "descifra" al sujeto.

6.Los vocabularios son deícticos: los significados dependen de quién los enuncia y del contexto en el cual lo hace; se basan en términos cuyo significado será accesible al observador gracias al contexto en el cual se utilizan, permiten darle contenido e reconocer la expresión deíctica (aquello que se dice, sin decir, pero lo dice por ser él quien lo dice, des- de dónde lo dice y por cómo lo dice) de su interlocutor.

En la práctica educativa como en los intercambios cotidianos partimos de que los sujetos en la interacción aprehende las "prohibiciones y la obligaciones" de la vida escolar, usan-aprenden los "procedimientos in- terpretativos" típicos del proceso de enseñanza-aprendizaje y otorgan y constituyen significados a las diversas acciones de quienes intervienen en éste. Esto es, la significación está en "medio" de la interacción, de la percepción y de la interpretación de objetos por parte de los sujetos, del sujeto frente a otro sujeto, de contenidos y conocimiento por parte de los aprendices.

El constitutivo central del significado es la vivencia del sujeto, "es dirigir la mirada a una vivencia que nos pertenece", lo que implica que se encuentra ya en el pasado, lo que Schutz denomina "contexto de significado o almacén de conocimiento". En tal sentido, la acción educativa se ubica en el terreno de las expresiones subjetivas entre asociados, paradójicamente, las actividades educativas se encuentran en un tipo de intersubjetividad donde las expresiones son objetivas, las relaciones son tales como si todos los sujetos compartieran costumbres, supuestos y conocimientos comunes. 


\section{La práctica reflexiva como base del desarrollo de las competencias docentes}

Desde la perspectiva de Philippe Perrenoud la constitución de una verdadera práctica reflexiva, demanda que se convierta en algo casi permanente y se inscriba dentro de una relación analítica con la acción, que se convierta en algo relativamente independiente de los obstáculos que aparecen o de las decepciones. "Una práctica reflexiva supone una postura, una forma de identidad o un habitus. Su realidad no se considera según el discurso o las intenciones, sino según el lugar, la naturaleza y las consecuencias de la reflexión en el ejercicio cotidiano del oficio, tanto en situación de crisis o de fracaso como a un ritmo normal de trabajo" (Perrenoud, 2004: 3).

Pierre Bourdieu, siguiendo a Aristóteles y a Santo Tomás definió el ha- bitus como la "gramática generativa" de las prácticas de unos actos, como un "sistema de disposiciones duraderas y que se pueden transponer que integra todas las experiencias pasadas y funciona en cada momento como una matriz de percepciones, apreciaciones y acciones, y posibilita el cumplimiento de tareas enormemente diferenciadas, gracias a las transferencias analógicas de esquemas que permiten resolver los problemas del mismo modo". Los esquemas de acción se pueden transponer, generalizar o diferenciar de una situación a la siguiente, es decir, lo que tienen en común con las diferentes repeticiones o aplicciones de la misma acción.

La práctica reflexiva postula que la acción es objeto de una representación; parte del supuesto de que el actor sabe lo que hace, y que por tanto, puede cuestionar los móviles, las modalidades y los efectos de su acción. Se da la posibilidad de que la existencia en el componente re- flexivo, asociado al pensamiento consciente, impidiera a Donald Shön reconocer abiertamente que toda acción compleja aunque, en apariencia, sea fundamentalmente lógica o técnica, únicamente es posible con base en funcionamientos inconscientes. En los oficios, los seres huma- nos no podemos pasar por alto la dimensión intersubjetiva de los mecanismos de negación o de rechazo, lo que constituye, el inconsciente práctico.

De la misma manera, existe una confluencia con los trabajos sobre la transferencia y la competencia, que hacen hincapié en los procesos de movilización de recursos cognitivos que permanecen en el inconscien- te, si no en la experiencia, por lo menos en el funcionamiento. Lo que sostiene Perrenoud al respecto, es que se trata del habitus que, "en- frentado a una situación lleva a cabo una serie de operaciones menta- les que garantizarán la identificación de los recursos pertinentes, su 
transposición eventual y su movilización orquestada para producir una acción adecuada. La alquimia es extraña porque la "gramática gene- rativa de las prácticas" no es una gramática formalizada" (Perrenoud,

2004: 38).

Los cuestionamientos que se formula el autor son básicos:

¿Acaso podemos reflexionar sobre nuestro propio habitus?

¿Qué tendremos que pagar por la labor de concienciación?

¿A dónde nos llevará esta reflexión?

¿Acaso da rienda suelta a los esquemas o se limita a alimentar situaciones de sorpresa, de vergüenza y de malestar?

Para dar respuesta, alude a la cultura individualista que Bourdieu deno- minó "la ilusión de la improvisación". Cada uno se imagina que inventa sus actos, sin percibir la trama constante de sus decisiones conscientes y todavía más, sus reacciones en caso de urgencia o rutinarias. Es di- fícil medir el carácter repetitivo de las propias acciones y reacciones, y sobre todo percibir de manera reiterada los efectos negativos de no hacer caso, asustar y ridiculizar a tal alumno o alumna, de formular consignas, de impedir que los aprendizajes reflexionen por sí mismos anticipando sus preguntas.

Existe una resistencia a la idea de que el habitus se mueve sin llegar a identificar los esquemas en juego, la necesidad de controlar nuestros actos, nos lleva a sobrestimar la parte consciente y racional de nuestros móviles y de nuestros actos. "Nuestra vida está hecha de repeticiones parciales. Las situaciones no varían hasta el punto de obligarnos, cada día, a inventar respuestas nuevas. La acción suele ser una repetición, con variaciones menores, de una conducta que ya se ha adoptado en una situación similar. La repetición, aunque sea menos apasionante que la invención permanente de la vida, está en el centro del trabajo y de toda la práctica, pese a que las microvariaciones exigen ajustes de los esquemas" (Perrenoud, 2004: 39).

En la medida que una postura práctica reflexiva tiene como objetivo regular la acción, no existe ninguna razón para que se detengan en el umbral menos consciente del habitus. En educación el practicante reflexivo es el símbolo de un acceso deseado al estatus de profesión docente de pleno derecho, que no está reconocido en el oficio de enseñante ni tampoco reivindicado por los sujetos que lo ejercen. Lo que conlleva la reflexibilidad es complejo, se trata, de acuerdo con Perrenoud:

- De extender las bases científicas de la práctica, allí donde existan, luchando contra la ignorancia todavía muy extendida de las ciencias humanas, de la psicología y aún más de las ciencias sociales.

- De no mistificarlas y de desarrollar formaciones que articulen racionalidad científica y práctica reflexiva; no como herramientas enemigas sino como 
dos caras de la misma moneda.

Lo anterior significa que la práctica educativa puede ser definida como "la acción dirigida por fines aparentemente conscientes", esto es, comprende acciones observables y tiene un fin planteado con anterioridad. No es pura acción, dado que ésta se encuentra ligada "al suceso que le da existencia, constituye una serie de vivencias que se forman en la conciencia individual de algún actor". En cambio la práctica educativa, se caracteriza por ser una acción intencional, que de acuerdo con José J. Brunner debe tener seis constitutivos: metas, punto de partida, punto de llegada, trayectoria entre ambos, se puede controlar y descomponer en etapas. En la práctica intencional es necesario que el saber qué, se transforme en saber cómo.

Desde el punto de vista aristotélico, la práctica se refiere a "una forma de vida característica - la bios praktikos - dedicada a la búsqueda del bien común. En este planteamiento se establece una diferenciación en- tre poiesis y praxis. El primer término se refiere a "la acción mental que consiste en hacer realidad algún producto o artefacto específico; el fin de la acción se conoce desde antes y se rige por la razón técnica", el segundo, también se dirige a "conseguir un fin, pero no busca producir un artefacto sino un bien moralmente valioso, no puede materializar- se, sólo puede hacerse, sólo puede realizarse a través de la acción y sólo puede existir en la acción misma" (Bazdresch, 2000:43). De la definición derivada del planteamiento de Aristóteles, Miguel Bazdresch caracteriza a la práctica educativa como "la acción intencional cuyo fin es educar, inseparable del medio que se usa y del bien que consigue" (Bazdresch, 2000:44).

A partir de la definición, el autor marca tres constitutivos o límites que se deben garantizar, si la intencionalidad es educativa: . educar supone un acto intencional del sujeto que va de no educado a educado; 2 . los actos intencionales suponen un proceso de concienciación, un proceso de conciencia reflexiva creciente acerca del objeto de conocimiento in- volucrado en la acción educativa; y 3 . la sensibilidad de observación y su relación con las decisiones inmediatas cotidianas del maestro, que pueden apuntalar o destruir un proceso educativo; sugiere buscar el constitutivo en la lógica de las acciones, realmente construida, reflexivamente concientizada y operacionalmente transformada. Para que la práctica educativa del docente universitario sea objeto de estudio, es indispensable ubicar el papel de los participantes en el proceso: educa- dor y educando.

Para Perreneud, la postura reflexiva pertenece al ámbito de las disposi- ciones interiorizadas, entre las que están las competencias, y también una relación reflexiva con el mundo y el saber, una curiosidad, una perspectiva distanciada, las actitudes y las necesidades de comprender. "Desarrollar una postura reflexiva significa formar habitus, fomentar la instauración de los esquemas reflexivos. Para Bourdieu, el habitus es nuestro sistema de estructuras de pensamiento, de percepción, de evaluación y de 
acción, la de nuestras prácticas. Nuestras acciones tienen

'memoria', que no existe en forma de representación y de saberes, sino de estructuras relativamente estables que nos permiten tratar un con- junto de objetos, de situaciones 0 de problemas" (Perrenoud, 2004:79). De aquí que, sea importante formar el habitus, como una mediación esencial entre los saberes y las situaciones que exigen una acción.

Esto es, no todas las conductas de los docentes son "automáticas", algunas recurren a los saberes que impulsan un desarrollo, el aprendizaje, la relación, el grupo, etc, pero es lo que permite activarlas en la situación. Para formar el habitus, es importante:

- Preguntarse para qué sirven los saberes en la acción, cuáles son mediaciones entre ellos y las situaciones.

- Aceptar la idea de que dicha mediación no está asegurada por otros saberes, sino por esquemas, que forman conjuntamente un habitus.

- Admitir que dicho habitus permite a menudo actuar "sin saberes" lo que significa "sin formación".

Desde la perspectiva de Perrenoud, "la formación del habitus debería constituir el proyecto de la institución completa, la cuestión principal de todos los docentes. Los docentes nos tendríamos que ocupar de la articulación de saberes y por el habitus; si los profesores utilizan poco los saberes didácticos, psicopedagógicos y psicológicos que han adquirido, es porque no logran conectarlos con la situaciones y por lo tanto, emplearlos con un buen criterio para actuar (Perrenoud, 2004:83). De acuerdo con el autor, el habitus procede de un inconsciente que Jean Piaget calificó de práctico: los conocimientos que el individuo posee de forma conceptualizada (no simbolizada), esto es, anterior a la transformación que caracteriza la concienciación; y el cómo llegar a la con- ciencia, mediante una conducta que constituye un verdadero trabajo cognitivo (no de terapia). Lo que destaca el autor es que el habitus es único y la conciencia de uno u otro esquema o conjunto de esquemas supone una labor mental que se distingue con base en la naturaleza de la acción. En otras palabras:

- Por un lado, la concienciación responde a la opacidad "funcional" de la práctica y a la dificultad de la explicitación o de la expresión con palabras.

- Por otro lado, los esquemas — de agresión, seducción, culpabilidad y angustia-, cuya concienciación se topa con los mecanismos activos de rechazo, con el presentimiento de que podrían desencadenar cambios 
irreversibles y sorprendentes.

Para el autor, el análisis de la práctica es posible cuando se mantiene la condición de ficción, "hablando de la práctica, no se habla del practicante y de su habitus" (Perrenoud, 2004: 60). Un problema adicional, es que no se dispone de las palabras y los conceptos que permiten hablar del habitus (como es de dominio público, el vocabulario psicoanalítico es más popular). La propuesta de Perrenoud es buscar métodos precisos que desarrollen una teoría del trabajo en general y del trabajo ensañante en particular, en sus dimensiones conscientes e inconscientes; $y$ 
su enfoque en particular, lo plantea en términos de habitus y de compe- tencias. Anota como importante en la profesionalización del desempeño docente: la especialización en el análisis de la práctica, en estudios de casos, en supervisión de periodos de prácticas, y en seguimientos de equipos y de proyectos, para llevar a cabo el trabajo de formador a partir de la práctica.

\section{REFLEXIONES FINALES}

Interesa destacar que la acción educativa puede no utilizar saberes ni razonamientos, sino que funciona con una especie de insconsciente práctico, cuyos mecanismos exactos no resultan difíciles de reconstruir. Trabajar sobre la práctica es, trabajar sobre un conjunto de acciones comparables y sobre lo que las sustenta y les asegura cierta invariabili- dad, determina lo siguiente:

- Saberes procedimentales (técnicas, métodos).

- Actitudes y rasgos de la personalidad (deseo de superarse, autocontrol, motivación, desapego o implicación).

- Diferentes componentes de la condición física y mental (fuerza y tono muscular, respiración, agudeza perceptiva, relajación, concentración).

- Esquemas de acción incorporados, que fundamentan las dimensiones estructurales de la acción, ya sea "concreta" (perceptiva, motriz), simbólica (actos de palabra) o puramente cognitiva (operaciones mentales).

Algunas de las condiciones mínimas, que Perrenoud ubica para el trabajo reflexivo sobre el habitus y esquemas incorporados en los docentes en educación superior son:

- Una cultura mínima en ciencias cognitivas, en psicoanálisis y en antropología de las prácticas.

- Una intención común y deliberada de trabajar en este nivel, y por tanto, de atribuir la prioridad a las estructuras invariables de la acción, sin detenerse en lo anecdótico (incluso se trata de un punto de partida obligatorio). 
- Una aplicación para describir la acción más que buscar inmediata- mente los móviles, una gran prudencia en las interpretaciones que la cargan de intencionalidad y sentido excesivos.

- Una ética coherente y una gran claridad conceptual, que permitan saber dónde está el límite entre un análisis de habitus invertido en la acción profesional y otros métodos como un psicoanálisis colectivo o una dinámica de grupos.

Después de realizar un análisis de las competencias docentes que pro- pone el autor, éstas son similares a las de Zabalza y Alfa Tuning para América Latina, como se puede observar en el cuadro de la página siguiente:

En la parte de instrumentación del diseño curricular de las competencias docentes, en un ejercicio de comparación realizado de 3 instituciones en el Distrito Federal que ofrecen Maestrías relacionadas con educación (véase Anexo ), se ubicó que las maestrías relacionadas con Educación y Ciencias de la Educación orientan sus objetivos hacia la docencia, la investigación, la planeación, la evaluación, y la gestión. Incluso uno de los ejes que definen a dos maestrías desde su nombre, es la investigación; Éstas son: Ciencias en Especialidad de Investigaciones Educativas, e Investigación y Desarrollo de la Educación, que tienen como objetivos:

- Dar una formación inicial que permita a sus egresados contribuir a la elaboración de este conocimiento de aportes teóricos y metodológicos de diversas disciplinas, en particular de la psicología, la lingüística, la sociología, la pedagogía, la historia y la antropología; mediante el análisis crítico y la investigación de los procesos educativos.

- Formar especialistas capaces de contribuir a interpretar y diseñar alternativas de solución a los problemas educativos de México y América Latina, especialmente los que afectan a los sectores socia- les menos favorecidos.

Las maestrías en Pedagogía y Pedagogía Universitaria plantean objeti- vos vinculados a la docencia y la investigación. Y la maestría en Desa- rrollo y Planeación de la Educación tiene como objetivos formar profe- sionales que: 


\begin{tabular}{|c|c|c|}
\hline Unión Europea (1999) & Philippe Perrenoud (1999) & Miguel Zabalza (2003) \\
\hline $\begin{array}{l}\text { Compromiso con el progreso } \\
\text { y logros de los educandos }\end{array}$ & $\begin{array}{l}\text { Organizar y animar } \\
\text { situaciones de aprendizaje }\end{array}$ & $\begin{array}{l}\text { Planificar el proceso de } \\
\text { enseñanza-aprendizaje }\end{array}$ \\
\hline $\begin{array}{l}\text { Capacidad en estrategias de } \\
\text { enseñanza-aprendizaje }\end{array}$ & $\begin{array}{l}\text { Gestionar la progresión } \\
\text { de los aprendizajes }\end{array}$ & $\begin{array}{l}\text { Seleccionar y preparar } \\
\text { contenidos profesionales }\end{array}$ \\
\hline $\begin{array}{l}\text { Capacidad para el } \\
\text { asesoramiento de educandos } \\
\text { y padres de familia }\end{array}$ & $\begin{array}{l}\text { Concebir y promover la } \\
\text { evolución de dispositivos } \\
\text { de diferenciación }\end{array}$ & $\begin{array}{l}\text { Ofrecer información y } \\
\text { explicaciones comprensibles } \\
\text { y bien organizadas } \\
\text { (competencia comunicativa) }\end{array}$ \\
\hline $\begin{array}{l}\text { Conocimiento de los temas } \\
\text { a ser enseñados }\end{array}$ & $\begin{array}{l}\text { Implicar a los alumnos en } \\
\text { sus aprendizajes y su trabajo }\end{array}$ & $\begin{array}{l}\text { Manejo de nuevas } \\
\text { tecnologías }\end{array}$ \\
\hline $\begin{array}{l}\text { Capacidad para } \\
\text { comunicarse con eficacia con } \\
\text { los grupos } \\
\text { v lncindividune }\end{array}$ & Trabajar en equipo & $\begin{array}{l}\text { Diseñar la metodología } \\
\text { y organizar las } \\
\text { actividades }\end{array}$ \\
\hline $\begin{array}{l}\text { Capacidad para crear un clima } \\
\text { conducente al aprendizaje }\end{array}$ & $\begin{array}{l}\text { Participar en la gestión } \\
\text { de la escuela }\end{array}$ & $\begin{array}{l}\text { Comunicarse-relacionarse } \\
\text { con los alumnos }\end{array}$ \\
\hline $\begin{array}{l}\text { Capacidad de hacer uso del } \\
\text { learning (aprendizaje electrónico) } \\
\text { y de integrarlo en los ambientes } \\
\text { de aprendizaje }\end{array}$ & $\begin{array}{l}\text { Informar e implicar } \\
\text { a los padres }\end{array}$ & Autorizar \\
\hline $\begin{array}{l}\text { Capacidad para manejar el } \\
\text { tiempo con efectividad }\end{array}$ & Utilizar nuevas tecnologías & Evaluar \\
\hline $\begin{array}{l}\text { Capacidad de evaluar su } \\
\text { propio desempeño }\end{array}$ & $\begin{array}{l}\text { Afrontar los deberes y los } \\
\text { dilemas éticos de la profesión }\end{array}$ & $\begin{array}{l}\text { Reflexionar e investigar } \\
\text { sobre la enseñanza }\end{array}$ \\
\hline $\begin{array}{l}\text { Conciencia de la necesidad del } \\
\text { desarrollo profesional continuo }\end{array}$ & Gestionar la propia formación & $\begin{array}{l}\text { Identificarse con la } \\
\text { institución y trabajar } \\
\text { en equipo }\end{array}$ \\
\hline $\begin{array}{l}\text { Capacidad para evaluar o } \\
\text { determinar los resultados de } \\
\text { aprendizaje y logros de los } \\
\text { educandos }\end{array}$ & & \\
\hline \multicolumn{3}{|l|}{$\begin{array}{l}\text { Capacidad para colaborar en la } \\
\text { resolución de problemas } \\
\text { Capacidad para responder a } \\
\text { las diversas necesidades de } \\
\text { los educandos }\end{array}$} \\
\hline \multicolumn{3}{|l|}{$\begin{array}{l}\text { Capacidad para mejorar el } \\
\text { ambiente de enseñanza- } \\
\text { aprendizaje }\end{array}$} \\
\hline $\begin{array}{l}\text { Capacidad para ajustar o } \\
\text { adecuar el plan de estudios } \\
\text { (curriculum) a contextos } \\
\text { educativos específicos }\end{array}$ & & \\
\hline
\end{tabular}


- Investiguen la problemática educativa en el México contemporáneo y su relación con los sectores social, productivo, político, administrativo y cultural.

- Planifiquen, sobre la base de la investigación y sus resultados, diseñen y pongan en marcha modelos de desarrollo educativo a nivel institucional, regional y/o nacional.

- Profundicen sus conocimientos en el sector educativo en sus diversas modalidades y contribuyan a la solución de sus problemas.

- Vinculen teorías y métodos en el análisis de los procesos educativos en relación con los demás procesos sociales y que dominen los aspectos estadísticos necesarios para el tratamiento de problemas relacionados con la planeación de la educación.

Lo anterior significa, que en el diseño curricular el tema de competencias docentes puede ser incorporado sin mucho problema. La pro- blemática principal se encuentra en la acción de los profesores. Como Perrenoud destaca es repetición, con variaciones menores que no nos obligan cada día a inventar respuestas nuevas. En este sentido, una postura y una práctica reflexivas tienen como objetivo regular la ac- ción, motivo por el cual no existe ninguna razón en la parte menos consciente del habitus. Para adaptar la acción a lo que tiene de trivial, es básico concienciar lo que tiene de trivial, porque ésta es la base de lo que pone en marcha los esquemas creados, dado que no existe prác- tica reflexiva sin el diálogo con el inconsciente práctico, pasar de una reflexión sobre la acción a un trabajo sobre la parte menos consciente del propio habitus "no es en absoluto fácil", pero es una de las formas que pensamos puede orientar la ruptura del habitus del homo academi- cus, el caso contrario, es la entrada a una cultura de mayor acento en la individualidad del trabajo académico.

Existe resistencia a pensar, no sólo que los profesores nos movemos por habitus sin tener consciencia de ello, y sobre todo, sin llegar a iden- tificar los esquemas en juego. "Nuestro deseo de control nos lleva a sobrestimar la parte consciente y racional en nuestros móviles y nues- tros actos". La consciencia choca con la opacidad de la propia acción y, todavía más, con los esquemas que la sostienen. Lo anterior, exige un trabajo intelectual que sólo es posible con la inversión de tiempo en ésta y con la adopción de un método y los medios apropiados (video, 
escritura o entrevistas de explicitación, audiograbaciones, etc.). La re- flexión sobre la acción, muestra la parte de nosotros que conocemos y amplía la reflexión a la parte inconsciente de nuestra acción. "Nadie desconoce que lo que hace es, en última instancia, la expresión de lo que es. Nadie está totalmente ciego a la importancia que revestirá po- der acceder a la gramática generativa de sus prácticas reflexionadas. Sin embargo, el practicante más lúcido prefiere cuestionarse sus sabe- res, su ideología y sus intenciones, más que sus esquemas inconscien- tes" (Perrenoud, 2004: 59)

Como conclusión final, podemos destacar que la práctica docente que pretende modificar el habitus de la educación formal e informal, pasa por lo que: la filosofía clásica griega, Antonio Gramsci, los anarquistas, Juan Amos Comenius, Jean Piaget, Lauwrence Kolberg, John Dewey, Pie- rre Bourdieu, Donald Shön y Philippe Perrenoud; han planteado desde diferentes acercamientos explicativos relacionados con la posibilidad de pensar en otras formas de organización social, esto es: la formación de la autonomía moral e intelectual de los seres humanos, no sólo de los profesores en todos los niveles educativos, que podría propiciar la integración de habitus flexibles y reflexivos en torno al comportamien- to individual y colectivo, y el impacto que tiene en el hecho de pensar en la educación como un bien público.

ANEXO
\begin{tabular}{|l|l|}
\hline \multicolumn{1}{|c|}{ Maestrías relacionadas con educación que se imparten en el D.F. } \\
\hline \multicolumn{1}{|c|}{ INSTITUCIÓN } & \multicolumn{1}{c|}{ MAESTRÍAS } \\
\hline 1. CUDEP & Educación (seis áreas terminales) \\
\hline 2. CINVESTAV & $\begin{array}{l}\text { Ciencias en la Especialidad } \\
\text { de investiga educativas }\end{array}$ \\
\hline 3. Universidad del Valle de México & Educación \\
\hline 4. Universidad de las Américas & Educación \\
\hline 5. Universidad Iberoamericana & $\begin{array}{l}\text { Investigación y Desarrollo } \\
\text { de la Educación }\end{array}$ \\
\hline 6. Universidad Intercontinental & Educación Superior \\
\hline 7. Universidad La Salle A.C. & Educación \\
\hline 8. UAM & $\begin{array}{l}\text { Desarrollo y Planeación de } \\
\text { la Educación }\end{array}$ \\
\hline 9. UNAM & $\begin{array}{l}\text { Docencia para la Educación } \\
\text { Media Superior } \\
\text { Pedagogía }\end{array}$ \\
\hline 10. Universidad Panamericana & Pedagogía \\
\hline 11. Universidad Pedagógica Nacional & Desarrollo Educativo-Escolarizada \\
\hline 12. Universidad del Pedregal & Ciencias de la Educación \\
\hline 13. Universidad Salesiana A.C. & Pedagogía Universitaria \\
\hline
\end{tabular}




\section{FUENTES DE CONSULTA}

- BAZDRESCH, Parada Miguel (2000). Vivir la Educación, transformar la práctica, Ed. Textos Educar-Educación Jalisco, México.

- BOURDIEU, Pierre ( 984) Homo Academicus, Les éditions de minuit, Francia.

- (2002)/Razones prácticas. Sobre la teoría de la acción, Ed. Anagrama, Barcelona, España.

- COMISIÓN EUROPEA (2006). ALFA - Tuning América Latina, Bélgica. [En línea] Disponible: http://www.tuning.unideusto.org/tuningal/index. php

- DE ZUBIRÍA, Samper Julián (2005). Competencias argumentativas. La visión desde la educación, Ed. Fundación Alberto Merani para el Desa- rrollo de la Inteligencia, Bogotá.

- EURYDICE (2002). Las competencias clave. Un concepto de expansión dentro de la ecuación general obligatoria, Ministerio de Educación, Cul- tura y Deporte, España.

- GRUNDY, Shirley (2007)/ "Killing me softly: the audit culture and the death of the participation in the modern university", ponencia presen- tada en el marco del Simposio Internacional de Investigación acción y educación en contextos de pobreza. Un homenaje a la vida y obra del Maestro Orlando Fals Borda, celebrado en Bogotá, Colombia.

- PERRENOUD, Philippe (2004). Desarrollar la práctica reflexiva en el oficio de enseñar: Profesionalización y razón pedagógica, Ed. Graó, Es- paña.

- (2000). Aprender en la escuela a través de proyectos: ¿por qué?,

¿cómo?, Revista de Tecnología Educativa, 4 (3), Santiago de Chile, pp.

$3-32$.

- (999). Construir competencias desde la Escuela, Dolmen Ediciones

S.A., Santiago de Chile.

- RYCHEN, Dominique Simona y Hersh, Salganik Laura (2004). Definir y seleccionar las competencias fundamentales para la vida, Ed. FCE, México.

- SABALZA, Miguel. Competencias docentes del profesorado universita- rio. Calidad y Desarrollo Profesional, Nancea S.A. de Ediciones, España, Segunda Edición, 2006.

- TORRES, Cárdenas Edgar et al (2002). El concepto de competencia. Una mirada Interdisciplinar, Ed. Sociedad Colombiana de Pedagogía, Bogo- tá, Colombia.

- TOBÓN, Sergio (2004). Formación basada en competencias, Ecoe Edi- ciones Ltda., Bogotá, Colombia. 